reality than of present misconceptions. But any forecasts are better than none, especially if their limitations are openly acknowledged. Probably the NSF will want to stick to comparatively modest forecasting until it has won a reputation for clairvoyance, but too much caution would be a mistake. Ironically, the evident value of this beginning will without question set off a clamour for a three or even a five year forecast as well.

There remains the question of how the forecasts can influence the willingness of the Federal Government to spend money on research and development. (Possibly, it is worth recalling that there are limits to the extent to which an agency like the NSF, itself dependent on the Government for funds, can forecast how much its own benefactor will be prepared to spend on agencies like itself.) Pressures may spring up in all directions, and the greatest danger is that Congress and the others holding purse strings may be mesmerized by the figures which have been produced for the various rates of growth. Experience in Britain as well as in the United States shows that treasuries find it almost irresistible to argue that the rates of growth for expenditure on science ought to be linked somehow to the rate of growth of the GNP or to the change of some other economic indicator. This is like putting the cart before the horse. Too much respect for the GNP as a universal yardstick is one way of bringing growth to a halt. In an expanding economy, it is inevitable that some things should grow more quickly than others, and it would be a great surprise if research and development were not among the most vigorous consumers of extra funds. If Congress wants to use the forecasts now produced as a guide to action in the next year or so, it should start from the forecasts of the skilled manpower that will be available in the year ahead and then reckon that enough money must be allocated for these people to be efficiently employed.

\section{WHERE ARE THE QUASARS?}

THE most distinctive property of the quasars is that the radiation from them is shifted enormously to the red, and any attempt to account for their existence must begin with that. But does the red-shift imply recession? And is an apparent recession of the quasars to be interpreted as participation in the general expansion of the universe? This is the train of thought which led, immediately after the discovery of the first of these objects, to the supposition that quasars are for one thing extremely far away, and therefore exceedingly powerful sources of radiation even by the yardsticks of astrophysies. But if red-shift implies distance, and if quasars are distributed more or less randomly throughout the universe, there should be a relationship between brightness and red-shift. The quasars with the biggest red-shifts should, on the average, be the faintest. That is how the argument began, but the problem of the quasars has so far been enormously complicated and confused by the failure to pick out anything like a significant correlation between the brightness of quasars and the red-shift of their radiation. A year ago, with an air of resignation, Hoyle and Burbidge wrote that "as new red-shift values become increasingly available, the plot of the observed quasi-stellar objects has assumed more and more the aspects of a scatter diagram" (Nature, 210, $1346 ; 1966)$. Although there have been several attempts to explain how a real correlation between brightness and red-shift may be obscured by irrelevancies, the absence of a correlation has been the chief reason why people have been energetically seeking ways of accounting for quasars which do not entail rapid recession at the edges of the universe.

It is too soon to know whether the problem will be simplified by the two articles on the red-shift relationship which appear on pages 917 and 919. Now that more data have accumulated, the beginnings of what seems to be a real correlation between brightness and red-shift does seem to be emerging. Horton and Daintree, writing from Jodrell Bank, argue that the relationship is more clearly apparent at higher radio frequencies than those used in earlier comparisons, and they claim that the most compact and the brightest of the quasars in their sample have a brightness and red-shift related by a simple curve not altogether different from the predictions of some cosmological models. In their view, the failure of all quasars to lie on the same smooth curve may be accounted for by processes such as internal absorption of radiation within particular objects. To them, red-shift implies great distance.

Although Longair and Scheuer agree that the newly accumulated data on quasars imply a significant relationship between red-shift and brightness, their interpretation is quite different. They argue that the observed brightness of a quasar with a pronounced red-shift is not itself a measure of the power emitted, but must be corrected to account for several complicating factors-the fact that a red-shift of any origin will reduce the energy of photons and the rate at which they reach an observer, for example. In other words, to them a faintly significant correlation between red-shift and brightness is not a sign that quasars are distant objects but rather a somewhat unsurprising happening which is entirely consistent with the view that the redshift of quasars has nothing to do with rapid recession or great distance. The most convineing part of what Longair and Scheuer have to say is based on an analysis of the optical brightness of a number of quasars. The difficulty, of course, is that their negative conclusion may not be valid for the data corresponding to the very high radio frequencies at which Horton and Daintree claim the relationship is most apparent. In other words, the two arguments are not necessarily in conflict. The immediate result, no doubt, will be a careful poring over data. The theoreticians anxious to get on with model building will have to wait a little longer. 